Check for updates

Cite this: Food Funct., 2021, 12, 6691

Received 29th March 2021,

Accepted 19th May 2021

DOI: $10.1039 / \mathrm{d} 1 \mathrm{fo} 00956 \mathrm{~g}$

rsc.li/food-function

\section{Gastrointestinal digestion of dietary advanced glycation endproducts increases their pro-inflammatory potential $\uparrow$}

\author{
T. van der Lugt, (D) *a,b M. F. Vrolijk, ${ }^{c}$ T. F. H. Bovee, ${ }^{d}$ S. P. J. van Leeuwen, ${ }^{d}$ \\ S. Vonsovic, ${ }^{d}$ A. Hamers, ${ }^{d}$ A. Opperhuizen ${ }^{a, b}$ and A. Bast ${ }^{a, c}$
}

\begin{abstract}
Thermal treatment of food products leads to the formation of dietary advanced glycation endproducts (dAGEs). It was previously shown that dAGEs induce TNF- $\alpha$ secretion in human macrophagelike cells. To what extent gastrointestinal digestion of dAGEs influences these pro-inflammatory effects and what the implications of these pro-inflammatory characteristics further down the human gastrointestinal tract are, are currently unknown. In one of our previous studies, dAGEs were digested using the TNO gastrolntestinal Model and analysed for dAGE quantity after digestion. In the current study both digested and undigested dAGEs were used to expose human macrophage-like cells, which were subsequently analysed for TNF- $\alpha$ secretion. In addition, the obtained digests were fractionated, and human macrophage-like cells were exposed to the different fractions to determine whether specific fractions induce TNF- $\alpha$ secretion. The results show that digested dAGEs have an increased pro-inflammatory effect on human macrophage-like cells compared to undigested dAGEs. This paper therefore shows that the digestion of food-components, and specifically dAGEs, plays an important role in determining their biological activity.
\end{abstract}

\section{Introduction}

During thermal food processing, reducing sugars and amino acids react via a series of non-enzymatic reactions, also known as the Maillard reaction. Among the molecules formed during this reaction are Amadori products, early glycation products, advanced glycation endproducts (AGEs), and melanoidins. In

\footnotetext{
${ }^{a}$ Department of Pharmacology and Toxicology, Maastricht University, Maastricht, The Netherlands. E-mail: t.vanderlugt@nvwa.nl

${ }^{b}$ Office for Risk Assessment and Research, Netherlands Food and Consumer Product Safety Authority (NVWA), Utrecht, The Netherlands

${ }^{c}$ Campus Venlo, Maastricht University, Venlo, The Netherlands

${ }^{d}$ Wageningen Food Safety Research, Wageningen University and Research,

Wageningen, The Netherlands

$\dagger$ Electronic supplementary information (ESI) available. See DOI: 10.1039/ d1fo00956g
}

previous studies, we have shown that dietary AGEs (dAGEs), when bound to proteins, have distinct pro-inflammatory effects in human macrophage-like (THP-1) cells. ${ }^{1,2}$ Since the protein binding appeared essential for this pro-inflammatory effect, it was investigated whether dAGEs were stable during digestion in the human intestinal tract by using the TNO GastroIntestinal Model (TIM-1). ${ }^{3}$ The results of that study revealed an intact protein/peptide binding of the dAGEs during digestion. The question remains if these digested dAGEs can still have pro-inflammatory effects on macrophagelike cells.

dAGEs exert their pro-inflammatory effects largely via activating the receptor for advanced glycation endproducts (RAGE). ${ }^{1,4,5}$ RAGE is a $35 \mathrm{kDa}$ transmembrane receptor and has a multitude of ligands. Evidence suggests that activation of RAGE is dependent on the molecular size of the ligand, which explains why mainly protein-bound AGEs seem to activate RAGE. This size-dependency is likely due to oligomerization of RAGE in the cell membrane. ${ }^{6}$ However, the specific recognition sites of RAGE, or the required ligand size for binding is currently unknown.

In the current study it was assessed whether digestion alters the pro-inflammatory potential of glycated casein (GC), a heated food-based matrix containing a diverse set of dAGEs. In addition, digested and undigested GC was fractionated by sizeexclusion chromatography (SEC) and the obtained fractions were also tested for pro-inflammatory effects in macrophagelike cells in order to determine if the pro-inflammatory effect is size-dependent.

\section{Materials and methods}

\subsection{Chemicals and reagents}

Casein from bovine milk, lactose monohydrate, $\mathrm{NaOH}$, sodium-phosphate, 2-mercaptoethanol, and thiazolyl blue tetrazolium bromide (MTT) were obtained from Sigma-Aldrich (Saint Louis, MO, USA). D-Glucose, foetal bovine serum (FBS), 
Dulbecco's Phospate-Buffered Saline (DPBS) were obtained from Gibco (Thermo Scientific, Waltham, MA, USA). Phorbol 12-myristate 13-acetate (PMA) was obtained from Sigma (Zwijndrecht, Netherlands).

\subsection{Preparation of glycated casein (GC) and unglycated casein (UC)}

Casein, glucose, and lactose, in the proportion of two times concentrated milk powder (22 mM glucose, $0.4 \mathrm{M}$ lactose, $20 \mathrm{~g}$ $\mathrm{L}^{-1}$ casein from bovine milk), were suspended in $50 \mathrm{mM}$ phosphate buffer $\mathrm{pH} 7.4$ and heated on a heating plate at $100{ }^{\circ} \mathrm{C}$ in an Erlenmeyer flask for $60 \mathrm{~min}$ to form GC. After $60 \mathrm{~min}$, the samples were cooled in ice water, aliquoted and stored at $-80{ }^{\circ} \mathrm{C}$.

Control samples of glucose, lactose and casein, in the same concentrations, were prepared without heating (unglycated casein (UC)) and were also immediately stored at $-80^{\circ} \mathrm{C}$. The levels of different dAGEs in GC are given in ESI Table S1. $\dagger$

\subsection{Digestion of GC and UC}

The GC and UC samples were digested using TIM-1 as described previously. ${ }^{3}$ In short, 300 grams of GC and UC were run through the model that comprises four connected glass compartments, representing the stomach, duodenum, jejunum, and ileum. In the model, the inner walls of the four glass compartments are squeezed, simulating peristalsis, and in each individual compartment the $\mathrm{pH}$ is measured continuously and automatically regulated by the 'secretion' of hydrochloric acid (gastric compartment) or sodium bicarbonate (intestinal compartments). Secretion fluids (electrolytes, enzymes, bile, and pancreatic juice) were adjusted to the average concentrations as described for adults after ingestion of a meal. Pancreatic output was simulated by secreting $10 \%$ pancreatin in the small intestinal electrolyte solution. Biliary output was simulated by secreting a $2 \%$ bile (porcine bile

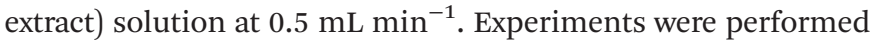
both in the gastric compartment only (first compartment representing the stomach), as well as in the complete model (all 4 compartments, representing stomach, duodenum, jejunum, and ileum). In the experiments in which only the gastric compartment was used, the duodenal compartment was only used for neutralization of the gastric efflux, without secretion of bile and pancreatin. This neutralization occurred before sampling. Concentrations of $N^{\varepsilon}$-carboxymethyl-lysine (CML), $N^{\varepsilon}$-carboxyethyl-lysine (CEL), glyoxal-derived hydroimidazolone-1 (G-H1), and methylglyoxal-derived hydroimidazolone-1 (MG-H1), were assessed by UPLC-MS/MS and are shown in ESI Table $\mathrm{S} 1 . \dagger$

\subsection{Determination of endotoxin in GC samples}

The presence of endotoxin in the GC samples from all digestion time points was assessed by the commercially available PYROGENT Gel Clot LAL Assay with $0.06 \mathrm{EU} \mathrm{mL}^{-1}$ sensitivity (Lonza, Basel, Switzerland). The assay was performed in accordance with the manufacturer's protocol using endotoxinfree dilutions and reagent tubes (Lonza, Basel, Switzerland).

\subsection{Size exclusion chromatography (SEC)}

Undigested and digested GC samples were fractionated using a SEC system consisting of an Agilent 1200 series LC-UV system (Santa Clara, CA, USA), equipped with a TSKgel SuperSW2000, $4.6 \times 300 \mathrm{~mm}, 4 \mu \mathrm{m}$ particles (TOSOH Bioscience, Griesheim (Darmstadt), Germany). The system was run isocratically with a SEC diluent of $200 \mathrm{mM}$ phosphate buffer ( $\mathrm{pH}$ 6.7) with a flow of $0.35 \mathrm{~mL} \mathrm{~min}^{-1}$ and the column was kept at room temperature. For each run, $100 \mu \mathrm{L}$ of undiluted sample was injected. The fractionation run lasted $20 \mathrm{~min}$ and fractions of $30 \mathrm{~s}$ (equalling $0.175 \mathrm{~mL}$ ) were collected between 5 and $14 \mathrm{~min}$. Protein size calibration was performed using a standard solution of 15-600 kDa protein mix consisting of thyroglobulin (640 kDa), $\gamma$-globulin (155 kDa), albumin (47 kDa), ribonuclease A (13.7 kDa) and $\rho$-aminobenzoic acid $(0.14 \mathrm{kDa})$ (Sigma-Aldrich, St Louis, Missouri, USA).

\subsection{Cell culture and exposure of macrophage-like cells (THP-1)}

THP-1 monocytes (ATCC, TIB-202), cultured in RPMI 1640 with L-glutamine, Hepes and phenol red (Gibco, Thermo Scientific, Waltham, MA, USA) supplemented with $10 \%(\mathrm{v} / \mathrm{v})$ foetal bovine serum (FBS), D-glucose $\left(4.5 \mathrm{~g} \mathrm{~L}^{-1}\right)$, sodium-pyruvate (1 $\mathrm{mM})$ and 2-mercaptoethanol $(50 \mu \mathrm{M})$, were seeded in a 96-wells plate at a cell density of 70000 cells per well. Differentiation into macrophage-like cells was induced by adding $200 \mathrm{nM}$ of PMA to the cell culture medium and culturing them for $72 \mathrm{~h}$. Macrophage-like cells were exposed to 1 and $10 \%(\mathrm{v} / \mathrm{v})$ of GC and UC in serum free and phenol-red free medium for $6 \mathrm{~h}$. Only endotoxin-free samples were used. Control samples included phenol-red free medium and 1 or 10\% DPBS.

To investigate the effects of the SEC fractions, the macrophage-like cells were exposed to $10 \%(\mathrm{v} / \mathrm{v})$ of unfractionated and fractionated sample, respectively. 10\% SEC diluent served as a control condition.

\subsection{Assessment of cell viability}

Cell viability macrophage-like cells was assessed using the MTT (3-(4,5-dimethylthiazol-2-yl)-2,5-diphenyltetrazolium bromide) assay. After removal of the cell supernatant, $100 \mu \mathrm{L}$ of MTT solution ( $0.5 \mathrm{mg} \mathrm{mL} \mathrm{m}^{-1}$ in DPBS) was added to each well. The plate was then incubated in the dark at $37^{\circ} \mathrm{C}$ for $1 \mathrm{~h}$. Subsequently, the MTT solution was removed from the wells and $100 \mu \mathrm{L}$ of DMSO was added to each well. After $10 \mathrm{~min}$ incubation at room temperature, absorbance was measured in a microplate reader (Bio-Rad, Hercules, CA, USA) at $\lambda=$ $540 \mathrm{~nm}$.

\subsection{Quantification of tumour necrosis factor (TNF)- $\alpha$ in cell culture supernatants}

Tumour necrosis factor (TNF)- $\alpha$ release in cell culture supernatants of macrophage-like cells was assessed by a commercially available ELISA kit (R\&D systems, MN, USA). The assay was performed in accordance to the manufacturer's protocol. 


\subsection{Determination of protein content}

Protein contents of TIM-1 digests of GC were assessed using the Pierce BCA Protein Assay Kit (Thermo Scientific, Waltham, MA, USA). The assay was performed in accordance to the manufacturer's protocol. Protein content of fractionated TIM-1 digests of GC was assessed using the NanoDrop (Isogen Life Science, Maarsen, The Netherlands).

\subsection{Statistics}

Experiments were performed in duplicate on different days with three repetitions using the average of the duplicates as one value. Data was analysed using GraphPad Prism software (v. 5.00, GraphPad Software, San Diego, CA, USA). Data obtained was tested for normality by using the D'Agostino and Pearson omnibus normality test. Due to a lack of normal distribution, data was analysed with the nonparametric KruskalWallis test followed by Dunn's Multiple Comparisons test or the Mann-Whitney U test to compare two sets of treatment. The significance level was set to $p<0.05$. The significance is indicated as ${ }^{*} p<0.05,{ }^{* *} p<0.01$, and ${ }^{* *} p<0.001$.

\section{Results}

\subsection{Pro-inflammatory effects of digested dAGEs on human macrophage-like cells}

To assess the pro-inflammatory potential of dAGEs after GI digestion, macrophage-like cells were exposed to a $1 \%(\mathrm{v} / \mathrm{v})$ solution of either TIM-1 digested GC or TIM-1 digested UC (as a control). Digested UC did not lead to any TNF- $\alpha$ secretion (data not shown). Since large dilutions of the food products occur during GI digestion, the TNF- $\alpha$ secretion was corrected for the protein concentration of the different samples and cell death (Fig. 1). Due to large deviations between experiments, the TNF- $\alpha$ secretion of the cells exposed to the digested GC

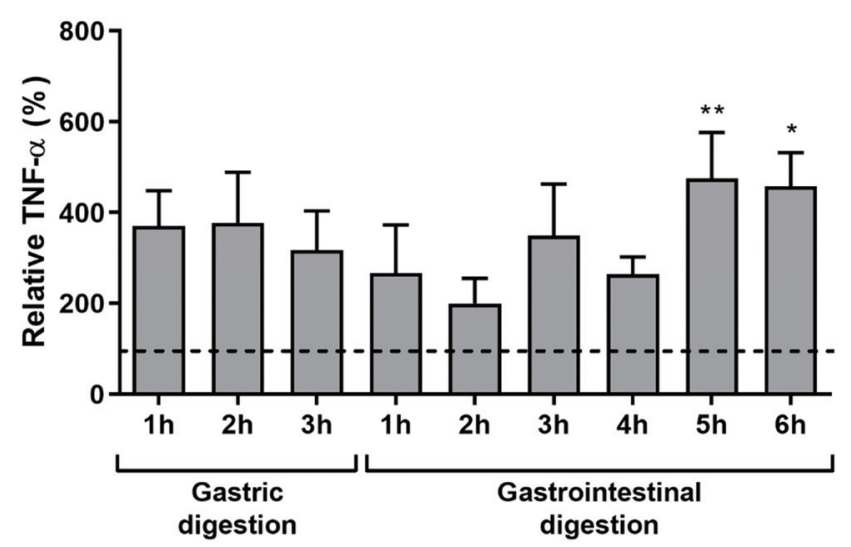

Fig. 1 TNF- $\alpha$ secretion of macrophage-like cells after $6 \mathrm{~h}$ exposure to digested GC samples (taken at different time points during digestion) relative to the undigested GC sample $(0 \mathrm{~h}$ digestion; dashed line at $100 \%$ ) measured by ELISA and corrected for cell death (MTT) and protein content of the dAGEs samples. Data are presented as mean $\pm \mathrm{SD}, n=3$. ${ }^{*}=p<0.05,{ }^{* *}=p<0.01$ compared to the undigested GC sample. was then expressed relative to the TNF- $\alpha$ secretion of the undigested GC sample (dashed line). The absolute TNF- $\alpha$ secretions are shown in ESI Fig. S1 $\uparrow$ and the undigested dAGEs led to 530 $\pm 252 \mathrm{pg} \mathrm{ml}{ }^{-1}$ TNF- $\alpha$ (mean \pm SD). Fig. 1 shows that gastric digestion of dAGEs led to an approximately $400 \%$ increase in TNF- $\alpha$ secretion and was not time dependent in the time window examined (1-3 h). Although the observed increases were not significant. Subsequent GI digestion significantly increased TNF- $\alpha$ secretion to over $400 \%$.

\subsection{SEC fractionation of undigested and digested dAGES}

In order to determine if the pro-inflammatory effect of dAGEs is size-dependent, the undigested and digested samples were fractionated by size-exclusion chromatography. The chromatogram of the protein standard control is shown in Fig. 2D. The TIM-1 control, i.e. reflecting the background signals caused by the enzymes and buffers used in the TIM-1 model, showed a chromatogram with a peak at approximately $640 \mathrm{kDa}$, some molecules smaller than $47 \mathrm{kDa}$ (including a clear peak below $14 \mathrm{kDa}$ ), and a peak below a molecule size of $0.14 \mathrm{kDa}$ (Fig. 2E). Separation of undigested dAGEs (Fig. 2A) revealed a large peak at approximately $6 \mathrm{~min}$ which corresponds to a protein size of approximately $640 \mathrm{kDa}$, a second peak at a size around $47 \mathrm{kDa}$, and several peaks smaller than $14 \mathrm{kDa}$. As expected, gastric digestion (Fig. 2B) led to a dramatic shift towards smaller molecule-sizes and the $640 \mathrm{kDa}$ peak at $6 \mathrm{~min}$ was reduced to a tenth (note the difference in $y$-axis scale). When comparing this peak to the same peak observed with the TIM-1 control (Fig. 2E), it can be concluded that this remaining peak results from molecules introduced and used by the TIM-1 model. Also the second peak at a size around $47 \mathrm{kDa}$ was strongly reduced after digestion (Fig. 2A and B). Additional peaks arise after longer elution times that were not present in either the undigested dAGEs nor in the TIM-1 control sample. Altogether, this indicates the presence of breakdown products of dAGEs after gastric digestion. After an additional $2 \mathrm{~h}$ of gastrointestinal digestion the peaks further shift to smaller sizes, most even below $14 \mathrm{kDa}$ (Fig. 2C).

\subsection{Pro-inflammatory effect of fractionated undigested dAGEs on human macrophage-like cells}

In order to investigate whether the pro-inflammatory effect of undigested dAGEs can be related to molecules of a particular size, macrophage-like cells were exposed to $10 \%(\mathrm{v} / \mathrm{v})$ dilutions of the different fractions collected after SEC (Fig. S2 $\dagger$ ). Fig. 3A displays the TNF- $\alpha$ concentrations in the cell supernatant induced by the different fractions. Absolute TNF- $\alpha$ concentrations were corrected for the background TNF- $\alpha$ secretion induced by only the SEC diluent per fraction. The unfractionated sample induced the highest TNF- $\alpha$ secretion. Exposing the cells to the first fraction did not lead to any TNF- $\alpha$ secretion, fraction 2 induced some TNF- $\alpha$ secretion $(3.4 \pm 1.9$ $\mathrm{ng} \mathrm{mL}^{-1}$ (mean $\left.\pm \mathrm{SD}\right)$ ) and fraction 3 induced $9.6 \pm 1.3 \mathrm{ng}$ $\mathrm{mL}^{-1}$ release of TNF- $\alpha$ from the macrophage-like cells. From fraction 4 to fraction $13, \mathrm{TNF}-\alpha$ secretion decreased, after 

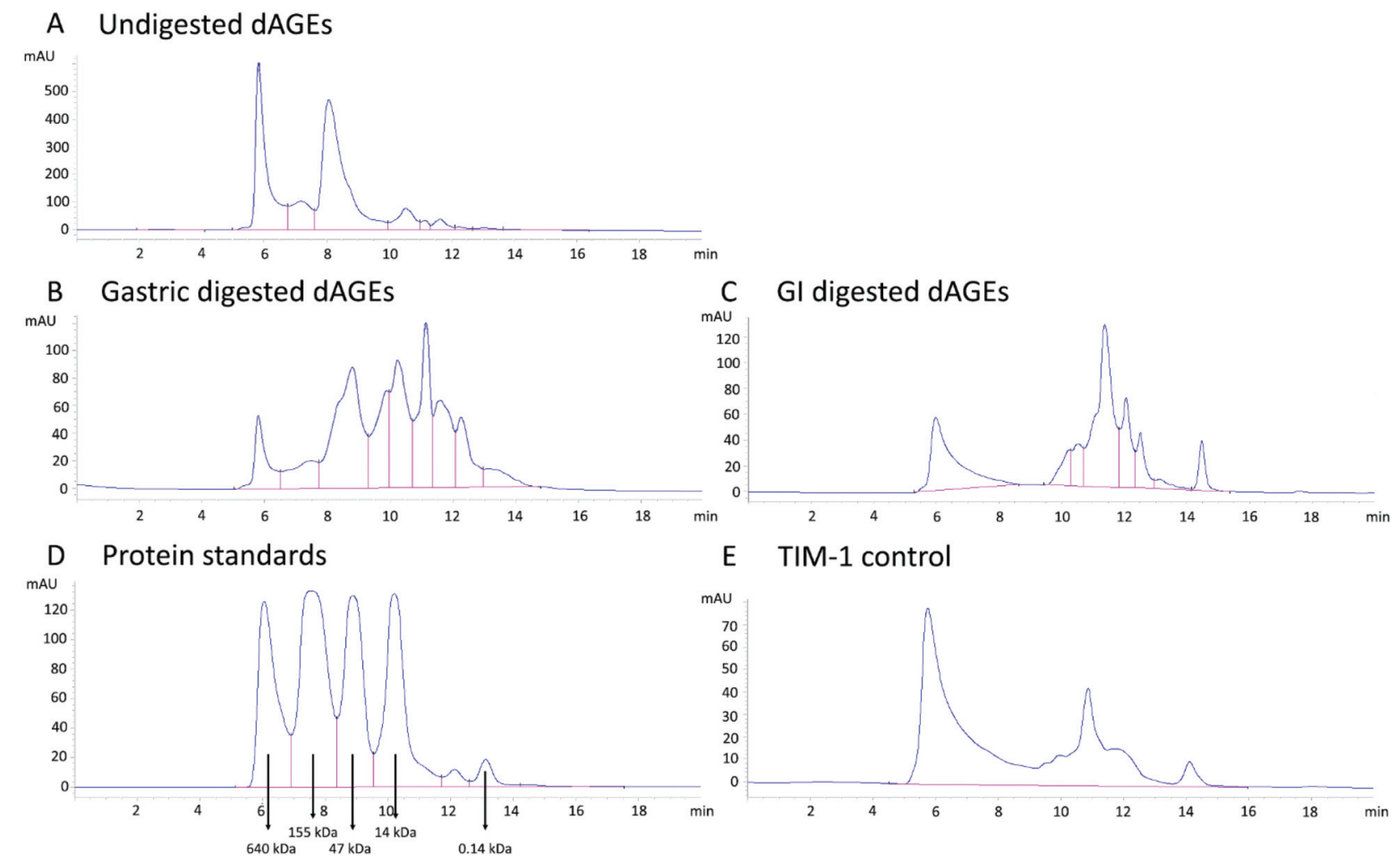

E TIM-1 control

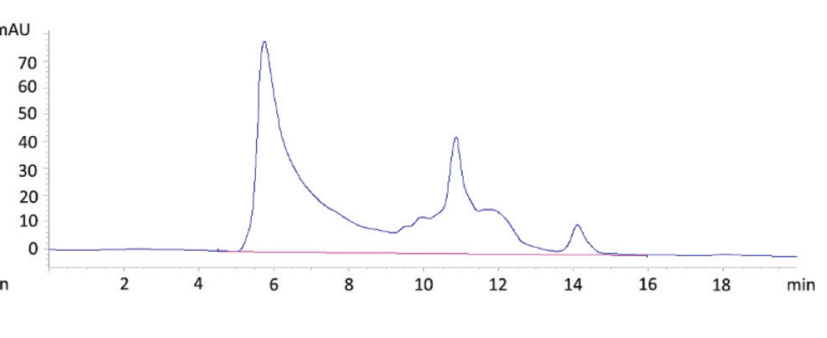

Fig. 2 Size-exclusion chromatograms showing the different fractions of (A) undigested dAGEs, (B) $2 \mathrm{~h}$ gastric digested dAGEs, (C) 2 h gastric digested and $2 \mathrm{~h}$ gastrointestinal digested dAGEs, (D) protein standards consisting of thyroglobulin (640 kDa), $\gamma$-globulin (155 kDa), albumin (47 kDa), ribonuclease $A(13.7 \mathrm{kDa})$ and $\rho$-aminobenzoic acid $(0.14 \mathrm{kDa})$ with indicated molecular weights, $(\mathrm{E}) \mathrm{TIM}-1 \mathrm{control}(2 \mathrm{~h}$ gastrointestinal digestion fluids). Milli absorbance units (mAU) are displayed on the $Y$-axis and elution time in min on the $X$-axis.

A

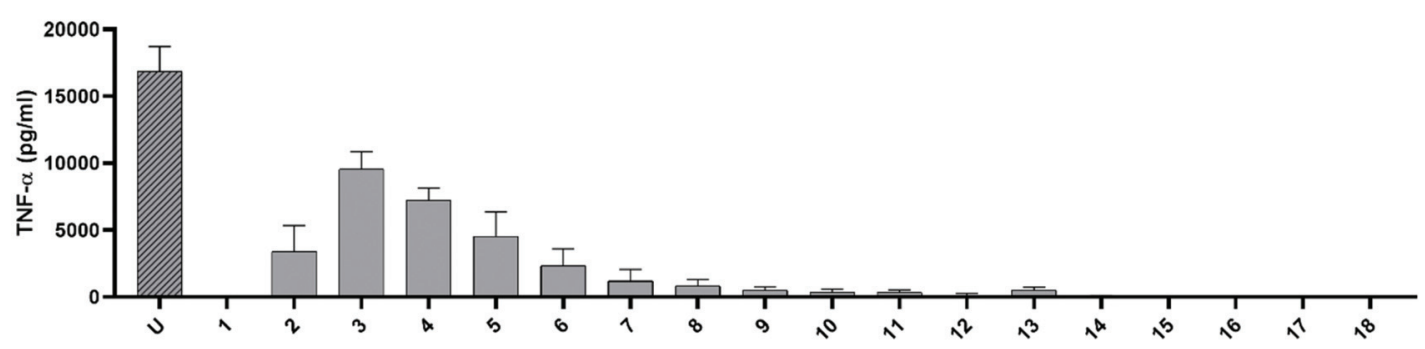

B

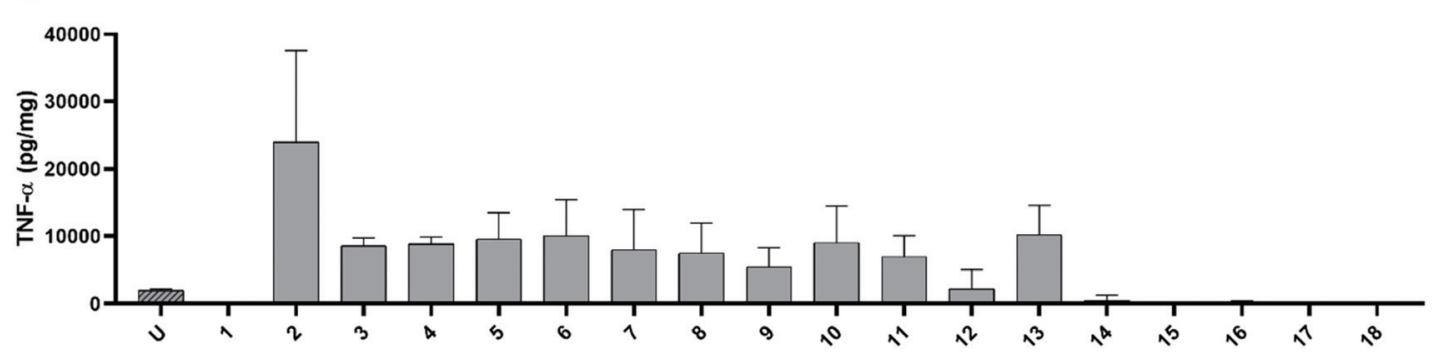

Fig. 3 TNF- $\alpha$ secretion macrophage-like cells measured by ELISA after $6 \mathrm{~h}$ exposure to the different fractions of undigested dAGEs separated according to molecular size (fraction 1 to 18). " $U$ " is the unfractionated sample. (A) Absolute concentration of TNF- $\alpha$ in pg $\mathrm{mL}^{-1}$ cell supernatant, (B) TNF- $\alpha$ secretion expressed in $\mathrm{pg} \mathrm{mg}^{-1}$ protein content of the undigested dAGE fractions. Data are presented as mean \pm SD, $n=3$. 
which no TNF- $\alpha$ secretion occurred anymore for the remaining fractions.

Since we have shown in earlier studies that predominantly the protein-bound dAGEs are responsible for the pro-inflammatory response of macrophages, the protein concentration of each fraction was determined and the TNF- $\alpha$ release per mg protein was calculated (Fig. 3B). Since the unfractionated dAGEs have a high protein content compared to the fractionated samples, it shows a relatively low TNF- $\alpha$ secretion per $\mathrm{mg}$ of protein compared to fractions 2 until 13. Fraction 2 greatly induced TNF- $\alpha$ secretion compared to both the unfractionated and the other fractions. Compared to the unfractionated sample this increase was $1190 \pm 670 \%$. Unfortunately, due to a large standard deviation this increase is not statistically significant. Fractions 3 up until 13 induced approximately the same amount of TNF- $\alpha$ secretion, up to $\pm 500 \%$ of the unfractionated sample. The fractions of the digested dAGEs were assessed in the same experiment, however the fractions of the TIM-1 control (without dAGEs), induced similar levels of TNF- $\alpha$ secretion as the fractions of digested dAGEs (data not shown). Indicating that it seems not to be possible to use all these bioassays (TIM-1, SEC, and THP-1 cell culture) in the same experiment.

\section{Discussion}

The pro-inflammatory effects of endogenous AGEs have been well documented. ${ }^{7-9}$ In contrast, the pro-inflammatory potential of dAGEs is hardly investigated and needs more attention. Our previous published study revealed that dAGEs, and specifically protein-bound dAGEs, also possess pro-inflammatory properties. ${ }^{1}$ However, before dAGEs can exert such effects in vivo in the intestines or systemically, they will have to survive digestion and possible inactivation by digestion in the GI tract. Previously we have shown that the protein-dAGE binding stays intact during GI digestion. ${ }^{3}$ To what extent the digestive processes influence the inflammatory effects of dAGEs is however not known yet. In the present study, the effect of GI digestion on the pro-inflammatory potential of dAGEs was assessed in human macrophage-like cells and compared to the pro-inflammatory effects of undigested dAGEs.

In order to mimic the digestion of dAGEs, GC, a heated food-based matrix with a variety of dAGEs, was run through the TIM-1 model. At several time points, samples were taken from the stomach and small intestinal compartment, which were administered to macrophage-like cells to measure the inflammatory effect. Our results show that digested dAGEs have a high pro-inflammatory potential (Fig. 1). Compared to undigested dAGEs, digested dAGEs appear to be approximately four times more pro-inflammatory. This raises the question of how this might be explained.

One of the possible mechanisms by which dAGEs could induce inflammation is via binding to, and subsequent activation of RAGE. RAGE is proposed to be a pattern recognition receptor and has many different ligands next to AGEs. Among these are proteins with high pro-inflammatory potential such as high mobility group box 1 (HMGB1), lipopolysaccharides (LPS), the signalling molecule lysophosphatidic acid, damageassociated molecular patterns, and advanced oxidation protein products. ${ }^{5,6}$ We have previously shown that blocking RAGE lowers the pro-inflammatory effects of dAGEs. ${ }^{1}$ Due to its nature as pattern recognition receptor, different sizes of protein-bound dAGEs might potentially interact differently with RAGE. A possible explanation of the different pro-inflammatory effects of digested dAGEs could therefore be the change in size of the dAGEs caused by the digestive processes. It is generally known that proteins are broken down into smaller peptides during digestion. As a consequence, smaller protein- or peptide-bound dAGE fractions could be formed in the GI tract. These changes could potentially change the interaction with RAGE and thus the pro-inflammatory characteristics of the dAGEs. Indeed, our results show that during digestion smaller sized proteins are being formed, as illustrated by multiple new peaks that were detected after gastric and smallintestinal GI digestion of GC (Fig. 2).

In order to determine a direct relationship between the size of dAGEs and their inflammatory potential, fractionation of undigested protein-bound dAGEs was performed by size exclusion and the pro-inflammatory effects of the obtained fractions were assessed. The obtained results show that especially fractions containing molecules larger than $640 \mathrm{kDa}$ induced high levels of TNF- $\alpha$ secretion (Fig. 3). Fractions smaller than $1 \mathrm{kDa}$ did not induce any TNF- $\alpha$ secretion. This indicates that especially the large molecules or aggregates are responsible for the pro-inflammatory effects of dAGEs. As stated before, RAGE is a pattern recognition receptor and oligomerization of RAGE on the cell surface is important for signal transduction, which might explain why specifically larger molecule structures bind and activate the receptor. ${ }^{6,10}$ An additional fractionation experiment was performed on digested protein-bound dAGEs and surprisingly, the fraction that has the highest inflammatory potential significantly decreased during digestion in our study. Hence, even though the larger fractions seem to be more pro-inflammatory, this does not explain why the digested dAGEs induce more TNF-a secretion than the undigested dAGEs.

The increased pro-inflammatory effects of dAGES after digestion seen in this study are thus not directly related to changes in size. However, to what extent digestion of dAGEs changes the binding to RAGE is currently unknown and therefore more research is needed to directly investigate whether our present results can be explained by RAGE activation. This could for instance be achieved by blocking RAGE on the cell surface or by assessing the different fractions in a RAGEbinding assay.

Another possible explanation of the increased pro-inflammatory potential of digested dAGEs may lie in the nature of the Maillard reaction. The Maillard reaction is an intricate reaction leading to different sub-sets of molecules. Not only AGEs are being formed, but also 1,2-dicarbonyls and melanoidins amongst others. These molecules may have different 
effects and could even interfere with each other. During digestion at $37{ }^{\circ} \mathrm{C}$, the Maillard reaction continues, leading to different sub-sets of molecules with different effects. It would be interesting to study whether the formation of different molecules during digestion causes the increased pro-inflammatory response after digestion.

In conclusion, our present study shows that digestion of dAGEs increases their pro-inflammatory potential. Importantly, the concentrations of proteins and carbohydrates used in this study are approximately $50 \times$ lower than would be encountered when drinking a large glass of reconstituted milk powder $(300 \mathrm{~mL})$. Unfortunately, our data do not provide a mechanistic explanation to why this is happening as the size of dAGEs does not directly explain the increased inflammatory potential of dAGEs after digestion. Future experiments should therefore be carried out to reveal the mechanisms involved in the observed effects. Our data do however prove that the digestion of food-components, and specifically dAGEs, plays an important role in determining their biological activity. The fractionated samples generally showed higher pro-inflammatory characteristics than the unfractionated sample. This is, in our eyes, a very important finding with implications for research into the biological activity of food-components.

\section{Author contributions}

Conceptualization: T. L., M. F., T. B., S. L; investigation: S. V., A. H., T. L. writing - original draft: T. L., M. F., writing - review \& editing: T. B., S. L., A. B., A. O.; supervision: A. B., A. O.

\section{Conflicts of interest}

There are no conflicts of interest to declare.

\section{References}

1 T. van der Lugt, A. Weseler, W. Gebbink, M. Vrolijk, A. Opperhuizen and A. Bast, Nutrients, 2018, 10, 1868.

2 T. van der Lugt, A. R. Weseler, M. F. Vrolijk, A. Opperhuizen and A. Bast, Nutrients, 2020, 12.

3 T. van der Lugt, K. Venema, S. van Leeuwen, M. F. Vrolijk, A. Opperhuizen and A. Bast, Food Funct., 2020, 11, 62976307.

4 K. Kierdorf and G. Fritz, J. Leukocyte Biol., 2013, 94, 55-68.

5 J. Xie, J. D. Mendez, V. Mendez-Valenzuela and M. M. Aguilar-Hernandez, Cell Signal, 2013, 25, 21852197.

6 J. Xie, S. Reverdatto, A. Frolov, R. Hoffmann, D. S. Burz and A. Shekhtman, J. Biol. Chem., 2008, 283, 27255-27269.

7 M. Pertynska-Marczewska, S. Kiriakidis, R. Wait, J. Beech, M. Feldmann and E. M. Paleolog, Cytokine, 2004, 28, 35-47. 8 K. Ikeda, T. Higashi, H. Sano, Y. Jinnouchi, M. Yoshida, T. Araki, S. Ueda and S. Horiuchi, Biochemistry, 1996, 35, 8075-8083.

9 G. Frati, L. Schirone, I. Chimenti, D. Yee, G. Biondi-Zoccai, M. Volpe and S. Sciarretta, Cardiovasc. Res., 2017, 113, 378388.

10 A. Moysa, D. Hammerschmid, R. H. Szczepanowski, F. Sobott and M. Dadlez, Sci. Rep., 2019, 9, 20332. 